\title{
The Role of Library Automation in Ensuring Distance Education to Confront the Pandemic COVID-19
}

\author{
Najat Wassila Belghanami \\ University Center Ali Kafi Tindouf, Algeria \\ wacila20006@hotmail.fr
}

\begin{abstract}
:
The Corona pandemic (Covid-19) has prompted governments of countries, after the suspension of studies, to resort to the distance education system, amid questions about the success of this experiment in light of a number of obstacles they are facing, which led to enhancing the automation of libraries, launching digital platforms and activating applications It is free for free access through digital platforms that allow examining educational content remotely in times of crisis to ensure the continuity of education. In this research paper, we refer to the role of automating libraries with various applications and functions, and then we review some models to ensure the continuity of learning through absolute distance education programs After the Covid-19 pandemic, through the launching platforms and virtual libraries that have undergone huge changes as a result of the rapid developments in the field of service management technology produced by information technology.
\end{abstract}

Keywords: Library Automation; Electronic Platforms; Electronic Applications; Virtual Libraries; Online Education. 


\title{
دور أتمتة المكتبات في ضمان التعليم عن بعد في مواجهة جائحة كوفيد 19
}

\author{
د.نجاة وسيلة بلغنامي \\ المركز الجامعي علي كافي تندوف، الجزائر
}

wacila20006@hotmail.fr

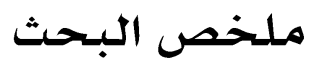

دفعت جائحة كورونا (كوفيد- 19 ) حكومات الدول بعد تعليق الدراسة، للجوء إلى نظام التعليم عن بعد، وسط تساؤلات عن مدى نجاح هذه التجربة في ظل وجود عدد من العقبات التي تواجهها.ما ادى الى تعزيز أتّتة المكتبات و اطلاق منصات رقمية و تفعيل تطبيقات بجانية للولوج المجاني من خلال منصات رقمية تسمح بتفحص المحتوى التعليمي عن بعد في اوقات الازمات لضمان استمرارية التعليم، عليه في هذه الورقة البحثية نقوم بالاشارة الم دور اتمتة المكتبات بمختلف تطبيقات ووظائفها ثم نقوم باستعر اض بعض النماذج لضمان استمرارية التعلم من خلال برامج التعليم عن بعد المطلقة بعد جائحة كوفيد 19 من خلال المنصات المطلقة والمكتبات الافتراضية التي خضعت إلى تغيرات ضخمة نتيجة التطورات السريعة في مجال التقنية الإدارية الخدماتية التي أفرزتها تكنولوجيا المعلومات.

الكلمات المفتاحية: أتهتة المكتبات، منصات الكترونية، تطبيقات الكترونية، مكتبات افتراضية، تعليهم عن بعد. 


\section{المقدمة}

بعد حالة اغلاق المكتبات حول العالم بسبب جائحة كوفيد 19 أدركت الحكومات نفسها بضرورة اتخاذ مناهج خختلفة، وأمرت أحيانًا بإغلاق جميع المؤسسات، وأخرى تشير إلى أن الحياة يجب أن تستمر كالمعتاد، والبعض الآخر ببساطة يترك القرارات لمديري المكتبات. أصبح لمدلول أتمتة المكتبة خيارا محتما بعدما كان محصورا في خو اص معينة منذ دخول الاتمتة إلى المكتبات مع مطلع عقد الستينات وحتى مطلع عقد التسعينات عبارة عن: قيام المكتبة بإنشاء نظام متكامل Integrated system يضم كافة فعاليات وأنشطة الأقسام من خلال قاعدة بيانات واحدة. من خلال نظم آلية تشغل من خلال حو اسيب كبيرة (Main Frame) أو متوسطة (Mini) أو حواسيب صغيرة (1) (1) (1) (1) حيث ساعدت هذه النظم التي تعتمد على بيانات في الغالب غير مدعمة بالصور أو الصوت أو الحركة. بالولوج المى محتوى المكتبات من خلال الشبكات في مواقع متقاربة و لا تعتمد كثيرا على الربط لإقامة مصادر معلومات إلكترونية في مناطق جغر افية بعيدة(2). خاصة بعد التطورات تكنولوجية الاتصالات التي شهدتها الشبكات وبالذات الإنترنت. فأصبحت الأتمتة في المكتبات أو أتمتة المكتبات تسمح بالإتاحة إلى مصادر المعلومات لم يعد مرتبطا بالجدران الأربعة لبناية المكتبة. بل تقدم خدماتها على مدار الساعة ولكل أرجاء المعمورة جعل توفر المعلومات حول العالم والوصول إليها أمرا في غاية البساطة والسهولة وكل مكتبة يمكن

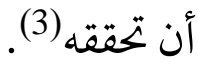
من هنا من خلال هذه المساهمة البحثية تتمحول اشكالية الدراسة في معرفة: ماهو دور اتمتة المكتبات لجمهورها قبل و بعد جائحة كوفيد 19 عليه من خلال هذه الورقة البحثية نحاول ان نقوم بالتعرض اولا الى دور ادارة المكتبات في الولوج الى قواعد البيانات وفقا على البيانات النصية (Textual) سواء كانت ثابثة ، متحركة و صوتية المعروفة بالـ(Media Multi). ثم مدى مساهمة انتشار الحوسبة في كل أنواع المكتبات و أنهاط الخدمات المحوسبة التي يستفيد منها جمهورها Super links, Link, ) من خلال التنقل الحر و السهل مابين قواعد بيانات عالمية و مواقع عن طريق الروابط 
(Hypertext من خلال بثها و اتاحتها للمستفيدين في كل أنحاء العالم عن طريق On Line Public Access) OPAC)، عدة منصات مطلقة و بر امج و تطبيقات بجانية مطلقة.

\section{أولا : ادارة المكتبـات ووظائفها:}

تقوم الادارة بدور المحرك الرئيسي في ادارة المكتبات حيث تمارس المكتبات الادارة لتحقق الهدف من وجودها من خلال العمليات المكتبية، فبدون الإدارة لن تكتمل حلقة الأداء التي توصل من خلالها المكتبات خدماتها إلى عموم المستفيدين. إضافة الى المهار ات الإشرافية اعتبارها أن القيمة المضافة لتطبيقاتها منذ اعداد ميز انيتها واختيارهم ، والمقر وتجهيزاته الى غاية استكملها كليا حيث تشمل إدارة المكتبة إدارة الموظفين العاملين فيها، إدارة الكتب مع المو اد المكتبية المختلفة التي تحتويها المكتبة. مما سبق من تعريفات للإدارة في المبحث السابق نستطيع أن نكون تعريفا شاملا لإدارة المكتبات على أنها عملية تنظيم الجهود، وتنسيق الموارد البشرية و التكنولوجية واستثمارها بأقصى درجة ممكنة من خلال التخطيط والتنظم والقيادة، الإشراف والرقابة، وذلك للحصول على أفضل النتائج و تحقيق الأهداف المطلوبة بأقل جهد، وقت مكنين (4). لذلك أصبح من واجب الإدارة و موظفيها تسخير كل الإمكانيات، الكفاءات من أجل تحقيق مناهج أغراضها في خدمة بجتمع المكتبة بتقديم كافة المعلومات لفم وتوجيهـم وإرشادهم إلى كل ما يوفر لهم المعرفة لذلك اضحى المدير المسؤول عن نجاح المدير ارتباط المرؤوسين بها يحقق انسجامهم معه في العمل (5). وبالتالي فإن الإدارة الفعالة للمكتبات تعتمد على تحديد أهداف المكتبة وإستخدام العدد المناسب والمؤهل للوظائف المكتبية والتوثيقية، فضلا عن التنسيق بين الوحدات والأقسام المختلفة وتوفير الميزانية الكافية. مما سبق يمكن القول ان إدارة المكتبات تعمل على تحقيق أهدافها وإعداد الكوادر البشرية اللازمة لتحقيق هذه الأهداف وذلك لخدمة المجتمع في مقابل الإمكانيات المتاحة من خلال العمليات الادارية التي تشكل النشاط الرئيسي لمديري المكتبات، كعملية ديناميكية و مستمرة(6). تتلخص وظائف العملية الإدارية بها يأتي: 


\section{أ- التخطيط:}

يتمثل في تحديد الأهداف العامة للمكتبة مسبقا على ضوء الموارد والإمكانيات المتاحة. في مختلف الدوائر والاقسام المختلفة، خلال فترة زمنية محددة، فالتخطيط في ادارة المكتبة القاعدة الرئيسية التي ترتكز عليها الجو انب الاخرى لذلك يتوجب على المكتبات ان تسعى الى تطوير ذاتها وتحديث معلو ماتها في خططها وبر ابجها المستقبلية لتعظيم رضى المستفيدين من خدماتها ، ومن أهم المجالات التي تحتويها ادارة المكتبات في التخطيط المستقبلي نجد الموارد المالية والبشرية ،استخدام التقنيات الحديثة، خدمات لجمهور القراء والمرتادين عليها وأنظمة الفهرسة والتصنيف (7).

\section{ب- التنظيه:}

هو تحديد و توزيع المسؤوليات و المهام و الصلاحيات والتقسيات الإدارية اللازمة، حسب الاختصاصات في تلك الأقسام بدقة بغرض تحقيق الأهداف. فهو يشكل عنصرا رئيسيا من عناصر إدارة المكتبات. لتصميم البنية الأساسية للمكتبة، وصف الأعمال و الأنشطة التي يجب أن تقوم بها التقسيهات الإدارية (الإدارات، الأقسام والشعب) اللازمة التي تستدعيها طبيعة العمل، كما يحدد العلاقات و أنماط الاتصال بينهم داخل المكتبة و خارجها بالاضافة المى تحديد مسؤوليات و واجبات لكل فرد من العاملين في المكتبة لتحقيق الأهداف المشتر كة(8).

\section{ج- التوظيف:}

هو شغل الوظائف المتو افرة في المكتبة بأشخاص أكفاء، قادرين علميا وفنيا وتقنيا على القيام بالعمل على أفضل وجه. عبارة عن اجتذاب المرشحين المناسبين لملء الشواغر للوظيفة المعلن عنها(9). لتزويد المكتبة بالكوادر البشرية اللازمة لإشغال الوظائف والعمليات الفنية وغير الفنية ولتقديم الخدمات المكتبية والمعلوماتية اللازمة. لذا ييب الاهتهام بالشخص المتقدم للوظيفة من حيث مؤهلاته وشخصيته، وتتم عملية اختيار 
الموظفين لإعطاء فرص متساوية للمتقدمين للوظيفة(10). حيث تحتاج المكتبات دائما إلى نوعين من الموظفين للقيام بأعلاما، خدماتها الفنية و العامة و غيرها من الأنشطة هما: فئة العاملين المتخصصين: هم الذي لديهم شهادات في علوم المكتبات أو التوثيق أو المعلومات من معاهد وجامعات معترف بها، وهؤلاء يعملون عادة في المجالات الإدارية والعمليات الفنية والخدمات والمعلوماتية المتقدمة(11). فئة العاملين غير المتخصصين: للقيام بالوظائف والخدمات غير الفنية وغير التخصصية والأعال الروتينية اليومية كالإعارة و غيرها(12).

\section{د- الإثـراف والتوجيـه :}

الإشراف هو المسؤولية عن عمل الآخرين الذين يقعون ضمن نطاق الإشراف. أما التوجيه فهو إرشاد المرؤوسين أثناء العمل من حيث إعطاؤهم التعليمات اللازمة للتنفيذ، وتفسير القضايا الغامضة لهم، وإرشادهم لكيفية التعامل مع ما قد يواجهونه من مشكلات أثناء العمل. التوجيه والاشراف وظيفتان متلازمتان تنطوي على كيفية تنفيذ الأعحال و إتمامها لتحقيق التنسيق من أجل تحقيق هدف مشترك (13). حيث يتعلق التوجيه في المكتبات بإرشاد المرؤوسين وتوجيهُم أثناء العمل، من حيث إعطائهم التوجيهات، الإرشادات، التعليمات، الأوامر اللازمة لحسن تنفيذ العمل و القيام به، مع ضمان فهمهم وقبولهم لها، بغرض تحقيق النتائج أو الأهداف المرجوة(14). ويقوم التوجيه السليم في المكتبات على مبدئين من مبادئ الإدارة هم) (15)

مبدأ تجانس الأهداف: يحقق تكامل و تجانس بين أهداف الأفر ادو أهداف المنظمة. مبدأ وحدة التوجيه (الرئاسة): أن ألا يكون الفرد مرؤوسا لأكثر من رئيس واحد حيث يقلل من المنازعات و الصراعات التنظيمية من خلال توجيه سليم يدل على الطريقة الصحيحة في العمل،

$$
\text { ويصحح الانحر افات والأخطاء (16). }
$$




\section{هـ- الرقابة:}

تعد الرقابة الحلقة الأخيرة من حلقات العملية الإدارية في المكتبات، فبعد أن يقوم المدير بالتخطيط و التنظيم و التوجيه يقوم بوظيفة الرقابة ليتأكد من أن التنفيذ يتم وفق ماهو خطط له، و ليثبت من دقة الاتجاه نحو الهدف، و من صحة السير نحوه(17). تعمل على ملاحظة تنفيذ الأععال في المكتبة، والتأكد من أنها تسير في الاتجاه الصحيح، وقياس ما تم إنجازه بالمقارنة مع ما حدد في الخطط من أهداف، ومحاولة اكتشاف أي انحراف عن هذه الأهداف ومعالجته بعد معرفة أسبابه، واتخاذ الإجراءات الكفيلة بمنع وقوعه مستقبلا، والتأكد من الوصول إلى النتائج المحددة وتحقيق الأهداف الموضوعة. من خلال اكتشاف الخطأ قبل وقوعه والعمل على منع وقوعه ما أمكن، و هذا ما يعرف بالرقابة الإيجابية الوقائية(18). أصبحت تقنية المعلومات و نظم التحكم الآلي بها يعرف بالأتمتة ضرورة من ضرورات تسيير ادارة المكتبات لما لـ تقدمه من منفعة لجحاعة الممارسة و المستفيدين منها، في هذا المبحث نظهر نشأة وتعريف أتمتة المكتبات في المطلب الأول من خلال استعراض مراحل استخدام الأتمتة في المكتبات، أما في المطلب الثاني خاص بمجالات عمليات استخدامها في المكتبات، وفي المطلب الأخير تناولنا تأثير أتمتة المكاتب على جماعة الممارسة وأصحاب المصلحة.

\section{ثانيا : مراحل استخدام الاتمتتة فِ المكتبات :} عرفت المكتبات منذ الستينات تغير ات ألحقت بها و على خدماتها من خلال تكنولو جيات المعلو مات و الوسائل الحديثة و بالضبط كانت بداية أتمتة المكتبات بالو لايات المتحدة الأمريكية في سنوات العشرينات من القرن الماضي .التي وجهت اهتمامها نحو تحسين الأعحال والأنشطة التي تشهد تكرارات عديدة ومتوالية على سبيل الذكر و ليس الحصر الفهرسة والإعارة لتقليل الضغط الدائم والمستمر على بنوك الإعارة، ومن ثمة تحسين الخدمات المقدمة للمستفيدين. من هنا تم التفكير في تطبيق أنظمة متداولة في ميادين الاقتصاد ، الصناعة واعتمادها داخل المكتبات وفق ما تقتضيه الحاجة . 
حيث تم اعتماد التقنية أول مرة صفائح معدنية محفورة فوقها رقم تسجيل القارئ واسمه، لتسهيل عملية الاعارة و مر اقبة الدخول إلى الأوعية ولكن تسجيل التأخيرات في الاسترجاع بقيت معقدة عليه. ثم تم ادخال تطبيق نظم الإعارة داخل المكتبات الذي تمخض عن التعاون مع شركات متخصصة في إنتاج الآلات و التقنيات الحديثة بإنشاء أنظمة معدلة من بينها "جيليود اليدوي" نظام بطاقة الجيب من إنتاج شركة جيليود الأمريكية. بحيث مكن هذا التطبيق من البحث عن بدائل أفضل لضبط عمليات الإعارة بالانتقال من استعمال الصفائح المعدنية التي تحدد تواريخ الإرجاع حسب فترات الإعارة المسموح بها، إلى بطاقة المستعير التي تستخدم بطاقتي التاريخ والإشعار الموجودتين في آخر الكتاب التي تمكن من طباعة رقم المستعير وتاريخ إرجاع المادة المعارة على كل منها التي من شأنها تحد من السجلات المتكررة و عدد العاملين المشاركين في علميات الإعارة(19)، ويهدفان إلى تسريع إجراءات الإعارة عند كل صباح. خلال سنوات الاربعينيات تم استحداث تقنية التمثيل التصوير التي تقوم بتصوير الكتاب المعار وكذلك بطاقة القارئ إضافة الى المستعير و الكتاب حيث يتم وضع بطاقة الاستعارة في محتوى الكتاب حيث تسلم المادة الى المستعير و بعد ارجاع المادة المعارة يتم سحب البطاقة منه بالرجوع الى الأفلام(20) هذه الاخيرة التي استغرقت مدة طويلة نوعا ما في عملية التركيب فتم تطوير التقنية باستعمال التسجيل الصوتي أثناء عملية الإعارة من خلال نظام الإعارة السمعية لحفظ الرقم التسلسلي لعملية الإعارة وتاريخ الإرجاع والمعلومات الأخرى عن المستعير والكتاب المعار، بالرغم من الإقلال من الإجهاد البصري وكذلك مضاعفة عدد الإعارات لم تعمر هذه التقنية طويلا. عليه ظهرت تقنية البطاقات المثبتة التي تسير المعلومات المقروهة آليا. و كل بطاقة تشكل ما يعرف حاليا بقاعدة بيانات او مصفوفة مقسمة إلى 80 عمودا مرقا كل عمود يحتوي على 12 تسجيل فقي (خطي) حيث يتطلب هذا النظام ملفين الأول يحمل ترقيم الكتاب والملف الثاني يخصص لبيانات القارئ(21).

في سنوات الخمسينات تزامنا مع ادخال الحوسبة للجيل الأول التي تعتمد بدرجة كبيرة على البطاقات المثقبة (22) داخل المكتبات بالضبط على مستوى الجامعات الأمريكية اقتصرت مهامها فقط على معالجة النصوص 
ومن ثمة اتضح أن الأجهزة المحوسبة تحدم الاحتياجات التي تلائم الأعهال التكرارية التي تستخدم نفس التسجيلة البيليوغر افية في عمليات الطلبات والتزويد والدفع والمحاسبة والإعلان عن الكتب الجلديدة وإعداد بطاقات الفهارس والتحكم في الإعارة والتعرف على الكتب المتأخرة(23). بالرغم من ذلك عملية إعداد الملفات التي تحتوي على تسجيلات البيانات كان يتطلب شهورا وكان البحث بواسطة الحاسوب آلات في المراحل الأولى يتم بالطريقة التتابعية و لا تضم جميع عمليات المكتبة في آن واحد بل تتعامل مع جزء واحد فقط من عملياتها مثل : الفهارس، الإعارة. في سنوات الستينات تم استخدام آليات جديدة في التسيير بالجامعات وبخاصة الدارسون في كليات العلوم والهندسة والتجارة تعتمد عمليات التخزين و الاسترجاع على الخط المباشر في بنوك المعلومات ومراصد البيانات العالمية والتجارية في أماكن متعددة(24) في هذا الصدد كان مكتبة (LAWRENCE) بجامعة كاليفورنيا أول من استعملت الحاسبات الآلية لهذا الغرض بمعالجة دورياتها حيث تم معالحة 160.000 تسجيلة في حوالي دقائق في الوقت الذي كان يؤدي فيه هذا العمل في حو الي 27 ساعة كاملة. تلتها في سنة 1961 المكتبة الطبية الوطنية (MEDICAL LIBRARYANALYSIS AND RETRIVAL) بمحاولة مكننة كل وظائف المكتبة و اجر اء عمليات البحث الوراقي "الببليوغرافي" بها في ذلك الفهرسة الآلية والإعارة الآلية كاول مكتبة تستخدم نظام متكامل سنة 1966 . بعد حوالي ربع قرن من استخدام الحاسب الالي تطورت أساليب استعال الحاسبات إلى ما يسمى بنظام المشاركة الذي يسمح من ربط أجهزة الإدخال والإخراج عبر حاسب مركزي كبير الذي يمكن من البحث في مراصد البيانات الأصلية (DIALOG)(25). وهنا ظهرت الببليوغرافيات المعالجة آليا و نشأت أكبر موزعات المعلو مات على (ARPANET) المعلومات عن طريق الشبكات بالرزم أو الحزم والذي أنشأت أول موزع على يد TECHNICAL وكان ذلك في (MASSACHUSETTS INSTITUTE OF TECHNOLOGY : MIT) (INFORMATION PROJECT TIP). بعد ذلك ظهرت الموزعات المتخصصة( DIALOG ). وفي عام 3719 تم انشاء قاعدة بيانات نيويورك تايمز ( DOWJONES) وفي سنة 1977 تم قاعدة بيانات قانونية (LEXIS) 
قاعدة (CNRS). هذه القو اعد يمكن الاتصال بها عن طريق شبكات المعلو مات للمؤسسات البحثية العاملة مع الحكومة الأمريكية، أما قواعد المعلومات الاقتصادية فنجدها استعملت شبكات التبادل بالرزم التي تملك مساحات هائلة للتخزين تحتوي ملفات بيانية هامة وتخزينها محليا ثم وضعها تحت تصرف المستفيد الذي يمكنه الإطلاع عليها باستع) (ON LINE PUBLIC ACCESS: OPAC) الخط المباشر. ومن طريقة اعتماد البحث عن الوثائق وإنتاج النشرات الببليوغرافية والكشافات تعدت الأنظمة المحسوبة بالاعتحاد على وسائل المعالجة الإلكترونية للمعلومات في: الإقتناء، التزويد، الفهرسة، التكشيف، إنشاء الملفات، البحث الوثائقي، إنتاج المنتوجات الوثائقية ، الإعارة، المراقبة والتسيير الإداري. عموما في سنوات الثمانينات كان استعمال الحاسب الآلي بالمكتبات الجامعية مقصورا على مهام التزويد والإقتناء، تسيير الإعارة وإعداد الفهارس علم) بأن هذه العملية تتم خطوة خطوة بعد دراسة كل عملية تفصيليا والتعرف على عدد وطبيعة واحتياجات مستخدمي المكتبة وكذلك الأخذ بأخذ بعين الإعتبار حجم المكتبة والموظفين والأجهزة والميزانية المطلوبة .

\section{ثالثا : عمليات و مجالات استخدلامـات الأتهتة فِِ المكتبات :} أصبحت المكتبات اليوم متو افقة مع ما أتمتة المكاتب والتقنية كشريك ونسيج يدخل في كافة تطبيقات المكتبات من خلال نظم إدارة المكتبات التي توفرها كضرورة حتمية، نظرا لما تقدمه من مزايا كبيرة تساعدها في تحسين مستوى الخدمات المكتبية وتقدميها في أقل وقت وجهد وتكلفة مككنة لتلبية احتياجات المستفيدين من خلال استخدام الحاسوب والبربجيات والتقنيات الحديثة الأخرى في انجاز أعملها المكتبية والتي تساعدها في السيطرة على الكم الهائل من المعلومات في خختلف ميادين المعرفة أهمها:

\section{أ- خلدمة التزويد والاقتنـاء الآلي:}

عملية توفير أو الحصول على الموارد المكتبية المختلفة والمناسبة للمكتبة و لمجتمع المستفيدين (26)، حيث يلعب الحاسوب و البربجيات و أدوات الاتصال دور مهم في تحضير الطلبات و تحويلها إلى الناشر مع الفواتير ورصد 
الحسابات و دفع جميع المستحقات(27) ومن الصفات الواجب توفرها في التزويد الإلكتروني يمكن ذكرها

$$
\text { كالآتي (28): }
$$

$$
\text { • القدرة على الطلب بالاتصال المباشر مع الموردين . }
$$

القدرة على البحث عن طريق نقط وصول خختلفة بالملف الببليوغر افي للمكتبة ذاتها لتعيين وضع أي مادة

\section{معينة.}

ه الوصول إلى البيانات لوضع جميع العناوين من الموردين لمعرفة عنوان معين غير منشور بعد أو أثناء ذلك.

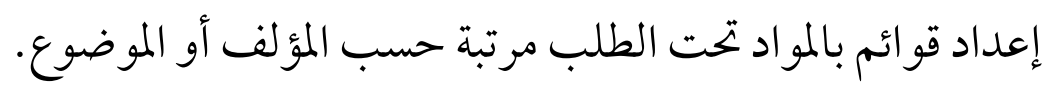

• إعداد قوائم بالمواد التي تم الحصول عليها و إرساها للجهات أو الأفراد المهتمين.

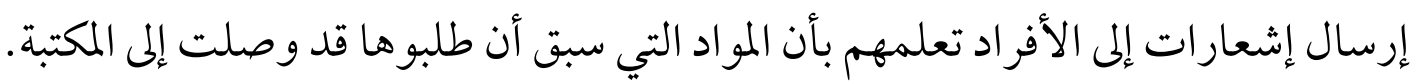

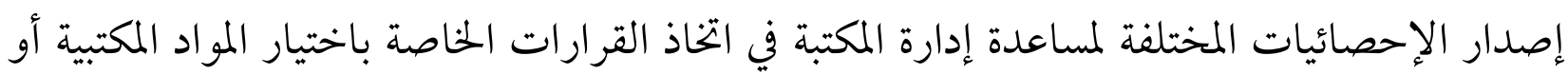

$$
\text { شر ائها و التحكم بالميزانية...إلخ. }
$$

القدرة على توفير معلومات مالية مفصلة جدا و بطرق مختلفة.

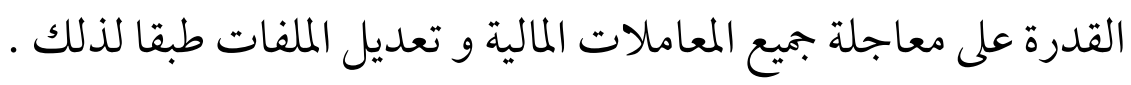

ومن التطورات الحديثة في مجال نظم التزويد المبنية على الحاسوب تمكن الناشرين وباعة الكتب من اتاحة طلب المواد المكتبية عن طريق محطة طرفية و بشكل مباشر في المكتبات دون اللجوء للمراسلة. ومن أمثلة هذه الخدمات خدمات أسكو Askew والنظم التعاونية مثل: ( OCLC (Online Computer Library Center)، بالإضافة إلى تسهيل عملية ملء البيانات و تخزينها في الحاسوب، الحصول على نسخ من طلبات الشراء التي ترسل إلى الموزعين أو الناشرين،إعداد التقارير المالية، الإحصائيات الإدارية، التقارير والوثائق الأخرى المطلوبة في عملية التزويد (29). 


\section{ب- خلدمة الفهرسلة الآلية:}

ساعد نظام الفهرسة المقروءة آليا (مارك) الى ارتقاء تنظيم السجلات الببليوغرافية وبثها في شكل مقروء آليا، باتباع التقنيات (القواعد) الدولية للفهرسة(30)، التي يمكن استرجاعها في أشكال يختلفة بها فيها الفهارس البطاقية و الميكروفلمية و الببليوغرافيات و نشر ات الإضافات و سجلات معروضة على شاشة الطرفيات، مما يتيح توفير البيانات الببليوغرافية في شكل منتجات تتماشى مع حاجة المستفيد و تبادل المعلومات الببليوغرافية مع المكتبات و مر اكز المعلومات من خلال (31): الطاقة التخزينية الهائلة والسرعة في استرجاع البيانات الببليوغرافية، ومن ثم تحديد مكان وجود الوعاء. إمكانية الوصول إلى المعلومات المطلوبة في الحال باستخدام وسائط نقل المعلومات المعاصرة كالأقمار الصناعية. كما أن للأقراص الضوئية و البصرية التي يتسع القرص الواحد منها مئات الآلاف من البطاقات الببليوغرافية التي تسترجع آليا، حيث تقدم إمكانية الا عحدودة لها للفهرس الموحد. كما حققت الفهارس الآلية قفزة نوعية من حيث عملية البحث للمستفيدين بالإضافة إلى ظهور العديد من

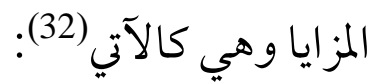

الفهرسة في عدة نقاط من المكتبة من خلال توفير المعلومات.

إمكانية الدمج بني عدة فهارس آلية من أجل تكوين فهرس موحد للمكتبات التي تعمل في إطار الشبكة (Réseau Régional Inter Bibliothèques RIBU ):مثلم) ما هو الحال بالنسبة لشبكة إمكانية الو صول عن بعد إلى فهرس المكتبة، وحتى طلب حجز الوثيقة عن بعد. تعدد معايير البحث مقارنة بالفهرس التقليدي، ففي الفهارس الآلية يمكن البحث بالعنوان، سنة النشر، نوع الوثيقة ....إلخ. إمكانية الدمج بني عدة معايير للبحث. سرعة تحيين الفهرس لمعرفة إن كانت الوثيقة المطلوبة متوفرة، في حالة ما إذا كان الفهرس مرتبطا بوحدة

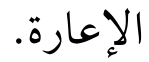


البحث بالفهرس الآلي يسمح بمعرفة وجود او عدم وجود الوثيقة دون بذل الكثير من الجها مقارنة

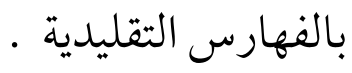

يسمح فهرس الاتصال المباشر للعامة ربط المستفيدين بفهارس المكتبات المتاحة عبر شبكة الإنترنت.

\section{ج- خدمة التصنيف الآلي:}

عبارة عن بناء قاعدة بيانات أساسية لتصنيف الموضوعات ضمن عناوين رئيسية وفرعية، حيث تضم وحدات متجانسة استنادا لمعيار محددا(33) على شكل تركيب هرمي يتدرج من العناوين الأعم والأكبر إلى الأخص والأصغر، بطريقة تتيح الوصول إليها بسهولة ويسر، بهدف تيسري الوصول إلى أي موضوع يريده

المستفيد(34). ويؤدي التصنيف الآلي مجموعة من الوظائف أهمها ما يأتي (35):

تنظيم مصادر المكتبة بقصد الاستفادة منها.

البحث عن المعلومات بسهولة ويسر وتجميع مصادر المعلومات الخاصة بالموضوع الواحد في مكان

$$
\text { واحد. }
$$

تسهيل عملية الجرد لمصادر ومقتنيات المكتبة.

تسهيل عملية الإعارة و التعرف على مواطن القوة و الضعف في مصادر المكتبة، وبالتالي يسهم في حفظ

$$
\text { التوازن بني مصادر المكتبة في المكتبات المختلفة. }
$$

\section{د- خدمة التكشيف الآلي:}

عملية إعداد الكشافات، أو مداخل الكشاف التي تؤدي للوصول إلى المعلو مات في ومصادرها، وتتضمن هذه العملية بايباز شديد: فحص الوثيقة، تحليل المحتوى وفقا للمعايير المحددة، تحديد مؤشرات المحتوى، إضافة مؤشرات المكان، و تجميع المداخل الناتجة في كل متماسك(36) ففي بجال التكشيف يقوم الحاسوب بفرز المداخل المحددة والمعدة يدويا ويبوبها ويعيد تركبيها وتحديثها وجمها، من ثم طباعتها، ورغم المحاولات 
لإعداد الكشافات الآلية إلى أنه لا يز ال للجهد البشري دور مهم يعتمد عليه بالكامل، ومن هذه الأنواع نذكر ما يأتي: - n

$$
\begin{aligned}
& \text { كشافات مبنية على كلمات مفتاحية. } \\
& \text { كشافات الإقتباس أو الاستشهاد(37). } \\
& \text { ومن فو ائد التكشيف الآلي في المكتبات نجد ما يأتي (38): } \\
& \text { الاختيار الدقيق للمصطلحات و التحكم في تشتت الموضوعات المتعلقة ببعضها. }
\end{aligned}
$$

التحكم في اللغة المستعملة في التعبير عن احتياجات المستفيدين من خلال ترجمة المصطلحات إلى لغات

\section{التكشيف الخاصة.}

$$
\begin{aligned}
& \text { حصر البحث من خلال توحيد لغات المساءلة . } \\
& \text { توفير الوقت والجهد في استرجاع المعلومات. } \\
& \text { سرعة الوصول للمعلومات. }
\end{aligned}
$$

ز زيادة التحكم في استرجاع الوثائق المختزنة وفقا لاحتياجات الباحث الموضوعية.

التعرف على محتوى الوثائق قبل الاطلاع عليها عليها بو اسطة الكشافات التحليلية.

\section{هـ- خدمة الاستخلاص الآلي:}

يعرف الاستخلاص بأنه التمثيل الدقيق و الموجز لمحتويات الوثيقة من خلال إعداد مستخلص بأسلوب شبيه بالوثيقة الأصلية، أي أن المستخلص يلخص المتينيل المحتويات الأساسية للتسجيلة الببليوغرافية ويعتبر كبديل

$$
\text { حقيقي للوثيقة الأصلية (39)، من أبرز فوائده: }
$$

الحصول على نصوص ووثائق جاهزة للطباعة الإلكترونية.

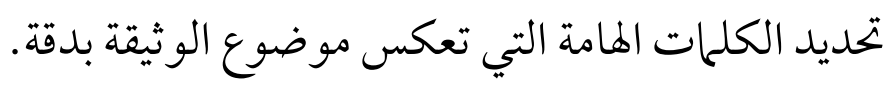

تمكين الحاسب الإلكتروني من قراءة الوثيقة وصياغة مستخلص لها باللغة الطبيعية.

السرعة و تقليل الجهاب في تحقيق هذه الخدمة (40). 


\section{و- خلدمة الاعارة الآلية:}

الاعارة هي بجموعة الاجراءات التي تمكن المكتبة من إتاحة الفرصة للمستفيدين للإفادة من مصادر المعلومات داخل مبناها أو خارجه لمدة زمنية معينة ووفق نظام محدد وضو ابط معينة تكفل المحافظة على تلك المصادر وإعادتها في الوقت المحدد، وتشمل أيضا عمليات الاعارة بين المكتبات ومراكز المعلومات بسرعة

$$
\text { ودقة(41). حيث تقوم بها يأتي: }
$$

التوثيق و الربط بني المادة و المستعير منها و التاريخ بشكل دقيق •

السيطرة على المواد المطلوبة للحجز.

$$
\text { إعداد إشعار ات عن الكتب التي انتهى تاريخ إرجاعها. }
$$

إجراء عملية التجديد و الحجز و الإعارة و الإرجاع و المطالبة آليا.

تحديد مكان وجود أي وثيقة و حالتها في الإعارة، الفهرسة، التجليد، إعارة تعاونية.

تقديم تقارير إحصائية لنشاط الإعارة في المكتبة و الذي يساعد الإدارة على سهولة تحليلها من أجل إعادة

بناء المجمو عات و زيادة عدد النسخ في بجال أو موضوع معين أو استبعاد ما يثبت عدم فائدة المستفيدين. ضبط عملية حجز الكتب المطلوبة من قبل المستفيدين عن طريق إرسال رسالة تنبيه إلى موظف الإعارة بأن الكتاب قد تم حجزه من قبل مستفيد آخر. إمكانية إعداد تقارير عن الكتب المتأخرة بشكل آلي (42).

\section{ز- خدمة الإحاطة الجاريـة الآلية:}

هي نظم استعراض أوعية معلومات أدب الموضوع المتاحة حديثا أو في كثير من الأحيان التسجيلات الببليوغر افية لأوعية المعلومات، و الاختيار من بينها الملائم لاحتياجات المستفيد و القيام بتسجيل تلك الأوعية وإرسالها إليهم، بمعنى جعل المستفيد على علم دائم بالجديد من خلال إحاطته علم) بظهور أوعية معلومات جديدة في بجال اهتماماته الموضوعية(43). حيث يمكن أداء هذه الخلدمة بطريقتين هما(44): إصدار تقارير ومطبوعات بأسماء المستفيدين، ويتم توزيع هذه التقارير عليهم حسب أسمائهم. 
ارسال القائمة إلى مجموعة من المستفيدين في مكان أو قسم واحد على أن تذكر أسماؤهم جميعا في القائمة مع وضع ملاحظة خاصة بتمرير تلك القائمة على الزملاء بالقسم في حال الانتهاء من الاطلاع عليها، و بالتالي توفير الوقت و التكاليف. وتكون الإحاطة الجارية على الوثائق من خلال عرض أشرطة بصرية على نهاية الحواسيب أو تقدميها إلى القراء على أقراص مرنة وطباعتها وعرضها في لوحة الإعلانات لتمكين المستفيدين من الاطلاع عليها، فخدمة الإحاطة الجارية تفيد بصفة عامة في تعريف المستفيد للتيارات الفكرية العلمية الحديثة(45)، فمن مزاياها ما

توحيد كثير من أنشطة المكتبات.

زيادة كمية الخدمات المقدمة و تحسين نوعيتها. توفري معلومات حديثة و آنية لمستخدمي المكتبات. إتاحة وسائل سهلة و سريعة للوصول إلى المعلومات. المرونة المعلوماتية مرتبطة باحتياجات المستفيدين ومتطلباتهم. زيادة الفعالية و تطوير الأداء في العمليات الفنية و الخدمات المعلوماتية. تقليص بعض الأعمال الروتينية و الاستفادة من وقت العاملين لأداء أعمال أخرى مهمة تقديم عروض تسويقية لخدمات المكتبات.

\section{ح- خدمة الببث الانتقائي الآلي:}

هي شكل من المساعدة البحثية المستمرة تقدم غالبا للباحثين الذين يستخدمون المكتبات الجامعية، وهي نوع من خدمة الإحاطة الجارية لأنها تجعل الباحثين محيطين بالتطورات الجارية التي تنعكس على الإنتاج الفكري في حقول تخصصاتهم (47).حيث يتم تجهيز المعلومات وتصنيفها ثم توجه إلى الباحثين على الخط المباشر بعد التعرف على مجال تخصص كل باحث وعنوان بريده الإلكتروني (48). ويعتمد البث الانتقائي الآلي عادة على جانبين هما(49): 


\section{بناء ملف اهتمام للمستفيذ يمثل توجهات حاجته إلى معلومات على شكل و اصفات.} مقارنة ملف الاهتمام هذا مع قاعدة البيانات المتوفرة في المكتبة، و المحدثة باستمرار، ضمن فترة زمنية محددة (شهريا، فصليا...الخ) أو بشكل آلي كلم) أضيفت لقاعدة البيانات تسجيلات جديدة، تعمل مقارنة مع ملف الاهتمام. حيث توفر خدمة البث الانتقائي مزايا عديدة نذكرمنها(50): تغطية شاملة من خلال الإحاطة الجارية لكل مستفيد على حدى. توفير وقت للمستفيد لاستيعاب الوثائق و المعلومات التي لا تناسبه . التعرف على الإصدارات الجديدة التي لها صلة بمواضيع اهتماماتهم، من خلال إدخال بيانات عن الباحثين في النظام الخاص بمواضيع بحوثهم و اهتماماتهم، في حالة الحصول على وثائق أو مقالات أو كتب لها صلة بهذه المو اضيع، توجه إليهم إشعارات تلقائية أو نسخ عن هذه الوثائق مباشرة، وهذه أرقى الخدمات التي يمكن للنظام تقدميها للمستفيدين.

\section{ط- الخدمة المرجعية الآلية:}

هي الخدمة التي تستقبل أسئلة المستفيدين عن طريق استحارة "الويب" أو البريد الإلكتروني أو عبر المحادثة على الشبكة. ليتولى بعد ذلك مكتبي المراجع استخدام هذه المدخلات من الأسئلة و الاستفسارات لبناء اجابة يتم استخدامها لتحقيق غرضين هما: ارسال الإجابة للمستفيد للرد على سؤاله، أما الغرض الثاني فيكمن في الاحتفاظ بالإجابات في قاعدة معرفية منظمة. حيث تعتمد الخدمة المرجعية الآلية على فكرتين هما: مساعدة الأفراد على ايجاد الجزء المقصود من المعلومة.

حرص المكتبات والمكتبيين على الإفادة من التقنيات الجلديدة لمساعدتهم في عملهم، وتعلمها وتقييمها والدمج بينها وبين عملهم اليومي، بغرض التوسع ليس فقط في قدراتهم المهنية، ولكن أيضا في درجة الاعتهاد على تلك التقنيات(51). من الأهداف التي يمكن أن تحققها هذه الخدمة في المكتبات نذكرها فيها يأتي (52): 
الإجابة على استفسارات و أسئلة المستفيدين عن معلومات معينة.

الإرشاد إلى المصادر التي تحتوي على المعلومات التي تتصل بأي مشكلة من مشكلات المستفيدين سواء

للبحث أو الدراسة.

تدريب المستفيدين على كيفية استخدام مصادر المعلومات بشكل عام و استخدام كتب المراجع بشكل

خاص للو صول على المعلومات و تقييمهاو استخدامها بكفاءة في عمليات البحث.

آليات ومقو مات تعزيز دور المكتبات في التعليم الالكتروني والتعليم عن بعد.

خدمات أتتمتة المكتبات لدعم التعليم عن بعد اثر جائحة كوفيد 19.

\section{رابعا : أتهتة المكتبات اثناء أزمة كوفيد 19 :}

بعد عرض بصفة جلية دور أتمتة المكتبات في تفعيل المعلوماتية على جماعات الملمارسة و اصحاب المصلحة نحاول ان نستعرض هنا ما حققته أتمتة المكتاب للتصدي لبوباء كورونا المستجد "كوفيد 19 " استجابة لمنظمة اليونسكو بغية بجابهة عن طريق شبكات الإنترنت الانقطاع عن الذهاب إلى المدرسة أو الجامعة منذ تعليق الدراسة اواخر شهر فبراير و بداية شهر مارس وتمديد التعليق حيث تم استجابة 61 بلدًا في أفريقيا وآسيا وأوروبا والشرق الأوسط وأمريكا الشمالية وأمريكا الجنوبية عن إغلاق المدارس والجامعات، أو قام بتنفيذ الإغلاق؛ إذ أغلق 39 بلدًا المدارس في جميع أنحائه، مما أثر على أكثر من 421.4 مليون طفل وشاب، كحا قام 14 بلدًا إضافيَّا بإغلاق المدارس في بعض المناطق لمنع انتشار الفيروس أو لاحتو ائه. وإذا ما لجأت هذه البلدان إلى إغلاق المدارس والجامعات على الصعيد الوطني، فسيضطرب تعليم أكثر من 500 مليون طفل وشاب

آخرين، وفق منظمة اليونسكو. و قد تم تسجيل (53): 1,184,126,508 الدارسون المتأثرون 67.6 إجمالي عدد الدارسين المسجِّلين 143 إغلاق في جميع أنحاء البلد 
أ- الآليـات المتخذة:

تعزيز استعمال الموارد السمعية والبصرية و التحول الى الاسلوب التفاعلي عن بعد بدلا من أسلوب "التلقين"

السماح بالدخول إلى المحتوى دون التوقف عند عتبات رائحة الأوراق. تطوير مناهج ابتكارية وبرامج دراسية ومسارات تعليمية بديلة وطرق التعليم العالي، وكل ذلك يمكن تيسيره عبر الإنترنت والتعليم عن بُعد والدورات القصيرة القائمة على المهارات. تفعيل مر اكز المعلومات مثل بنوك المعلومات. الاشتراك المجاني لكل الطلاب كوسيلة للتعلم عن بعد. أجبرت المكتبات على الإغلاق على سبيل المثال بـبعض حالات الاغلاق المسجلة : في غضون ذلك ، تأثر مكتبات المدارس في 177 دولة بإغلاق جميع المؤسسات التعليمية ، بينما في مدارس أخرى، تم إغلاق بعض المدارس على الأقل، وفقًا للأرقام الواردة من اليونسكو. وفي العديد منها، يتم إغلاق مكتبات الجامعة أيضًا. كما أغلقت المكتبات الوطنية أبو ابها للجمهور في ألبانيا، الجزائر، أندورا، أنتيغوا وبربودا، الأرجنتين، أستراليا، النمسا، أذربيجان، جزر البهاما، بنغلاديش، برمودا، بلجيكا، بوليفيا، البوسنة وهرسيغوفينا، البرازيل، بلغاريا كابو فيردي، كندا، شيلي، الصين، كولومبيا، جزر كوك، كوستاريكا، كوبا، قبرص،، كولومبيا، الدانمرك (مع خطط لفتح أبوابها ابتداءا من 10 مايو ) ، جمهورية الدومينيكان، الإكو ادور، مصر، إستونيا، جزر فارو، فيجي، فنلندا، فرنسا، جورجيا، اليونان، غرينلاند، غواتيهالا، غينيا بيساو، الكرسي الرسولي، المجر، أيسلندا، الهند، إندونيسيا، إيران، أيرلندا، جزيرة مان، إيطاليا، جامايكا، اليابان، كازاخستان، كينيا، قيرغيزستان، لاتفيا، ليختنشتاين، ليتوانيا، لوكسمبورغ، وماليزيا، والمالديف، ومالطا، وموريشيوس، والمكسيك، ومولدوفا، وموناكو، ومنغوليا، والمغرب، وناميبيا، وهولندا، وكاليدونيا الجلديدة، ونيوزيلندا، وشمال مقدونيا، والنرويج، وبنها، وباراغواي، وبيرو، والفلبين، وبولندا، والبرتغال، وقطر، وجمهورية كوريا، رومانيا، روسيا، سان مارينو، المملكة 
العربية السعودية، سنغافورة، سلوفاكيا، سلوفينيا، جنوب أفريقيا، إسبانيا، سانت لوسيا، سويسرا، تايلند، ترينيداد وتوباغو، تونس، تركيا، أوغندا، أوكرانيا، المملكة المتحدة، الولايات المتحدة الأمريكية، أوروغو اي. أعيد فتح المكتبات الوطنية في كرواتيا وتشيكيا وألمانيا وصربيا بصورة محدودة.

\section{ب- نهاذج عن بعض المنصدات المطلقة(54)؛}

منصة إدمودو (Edmodo)، وهي منصة اجتماعية بجانية توفر للمعلمين والطلاب بيئةً آمنةً للاتصال والتعاون، وتبادل المحتوى التعليمي وتطبيقاته الرقمية، إضافة إلى الواجبات المنزلية والدرجات

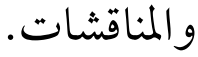

منصة( ONEFD ) كيوفر المكتب الوطني للتعليم والتدريب عن بعد (المكتب الوطني للتعليم والتكوين عن بعد) التابع لوزارة التربية والتعليم منصات تعليمية عبر الإنترنت في مواضيع مختلفة لجميع

$$
\text { المستويات الأكاديمية. ( الجزائر ). }
$$

منصة (EduNET ) ، يوفر خدمات تعليمية نختلفة لجميع مستويات المدرسة ويتيح التو اصل بين أعضاء

$$
\text { هيئة التدريس والطلاب وأولياء الأمور. ( البحرين). }
$$

منصة ( MOE E-Learning )، البوابة الإلكترونية الرسمية لوزارة التربية والتعليم ، بـا في ذلك البرامج التفاعلية يو ميا عبر (Edmodo) ،ووالدروس الافتر اضية والكتب الإلكترونية.( مصر). وضع منصتين لكل مستويات التعليم باسم ( Newton ) نيوتن و( parwarda-e)، بالإضافة إلى قناة تعليمية على موقع يوتيوب - محاضرات التليفزيون التربوي لجميع المستويات التي يقدمها الأساتذة والمديرية العامة للمناهج والتي تنتجها مديرية التلفزيون التربوي.( العراق). أطلقت منصتين، باسم "درسك 1" و"درسك 2"، تستهدفان كل مستويات الصفوف الدراسية في التعليم النظامي .عبر البوابة الرسمية للتعلم الإلكتروني في الأردن ، بها في ذلك دروس الفيديو لطلاب المدارس الابتدائية و الثانوية. بالاضافة الى ( Noorspace ) نظام إلكتروني موحد لجميع المدارس للتو اصل 
وتنظيم العمل بين المدارس والمعلمين والطلاب وأولياء الأمور من خلال بوابة إلكترونية يغذيها نظام إدارة المعلومات المدرسية الأردني بالتعاون مع مستودع الموارد التعليمية للمعلمين. (الأردن). منصة (MOE video channel) اضافة إلى قناة خخصصة على ( YouTube) تعرض دروسًا في الفيديو

$$
\text { ومؤتمرات من المعلمين. ( الكويت). }
$$

وزارة التربية والتعليم العالي أطلقت تطبيق التعلم الرسمي لوزارة التربية والتعليم العالي مع معلومات للمعلمين و العاملين في ججال التعليم.( لبنان).

$$
\text { قناة ليبيا الأحرار تبث وزارة التربية دروساً عبر قناة الأحرار للصفوف المختلفة.( ليبيا) }
$$

راديو موريتاني و ( TVM ) أنشأت وزارة التعليم الأساسي وإصلاح التعليم الوطني برنابجا تجريبيا

$$
\text { للتدريب عن بعد عبر وسائل الإعلام العامة لصالح طلاب الصف السادس. (موريتانيا). }
$$

منصة (TaalimTice) ) جموعة من مقاطع الفيديو حسب مجال الموضوع مقدمة من قبل وزارة التربية

$$
\text { و التعليم.( المغرب). }
$$

عمان مباشر والقنوات الفضائية الثقافية العمانية - القنوات التلفزيونية تنشر دروس التعليم عن بعد لطلاب الصف 11 و 12 ، وفقًا لجدول زمني محدد لجميع المو اد. سلطنة عمان)

قناة وزارة التعليم - بجموعة من 1700 درس عبر الإنترنت يتم تحميلها باستمرار إلى قناة (YouTube)

$$
\text { التابعة للوزارة مما يسمح للطلاب بمتابعة دروسهم عن بعد.( دولة قطر ). }
$$

أطلقت منصة وطنية عبر الإنترنت تستهدف 6 مليون تلميذ .البوابة الإلكترونية الوطنية (Ien ) هي بوابة التعلم الإلكترونية الرسمية لوزارة التربية والتعليم مع المواد الدراسية للصفوف من مرحلة ما قبل المرحلة الابتدائية وحتى المرحلة الثانوية.( المملكة العربية السعودية). منصة (ECE) منصة تعليمية لتعليم الطفولة المبكرة مع دروس فيديو ومواد أخرى. اضافتا المى المنصة التعليمية السورية - المنصة الرسمية لوزارة التربية والتعليم التي تحتوي على فيديو تعليمي تفاعلي ومواد 
سمعية لطلاب المدارس الابتدائية والثانوية. وقناة يوتيوب - قناة يوتيوب التابعة لوزارة التربية والتعليم

مع مستودع للصفوف على الإنترنت.( الجممهورية العربية السورية). مكتبة وزارة التربية عبارة عن منصة أنشأتها وزارة التربية و التعليم تتيح للمعلمين و الطلاب الاطلاع على مناهج التعلم و التفاعل معها إلكترونياً وتنزيل نسخ إلكترونية من الكتب.( الإمارات العربية المتحدة) أمثلة عن بعض التطبيقات المستخدمة : لتعزيز نظم إدارة التعلّم الرقمي (55):

وضعت المنظمة مجموعة من البرامج التي تساعد على التعلم عن بعد، ومنها تطبيق (Black Board) ، "بلاك بورد" تطبيق يعتمد على تصميم المقررات والمهات والواجبات والاختبارات وتصحيحها إليكترونيّا، والتواصل مع الطلاب من خلال بيئة افتراضية وتطبيقات يتم تحميلها عن طريق الهواتف

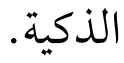
تطبيق "إدراك"، المعني بتعليم اللغة العربية عبر الإنترنت ويوفر موارد تعليمية هيئة التدريس والدارسين. تطبيق (Google Classroom)، "جوجل كلاسروم " الذي يسهّل التواصل بين المعلمين والطلاب سواء داخل المدرسة أو خارجها. تطبيق (seesaw) "سي سو"، وهو تطبيق رقمي يساعد الطلاب على توثيق ما يتعلمونه في المدرسة وتقاسمه مع المعلمين وأولياء الأمور وزملاء الدراسة، وحتى في العالم. تطبيق (Mindspark) )، الذي يعتمد على نظام تعليمي تكيُّي عبر الإنترنت، يساعد الطلاب على ممارسة الرياضيات وتعلمها. تطبيق سينشتري تك أو تقنية القرن، يقدم مسارات للتعلّم الشخصي بو اسطة دروس مصغرة تعمل على سدّ الثغرات المعرفية، وتنمي روح التحدي لدى الطلاب وتعزز الذاكرة الطويلة الأمد. 
تطبيق موودل: منصة للتعلّم المفتوح، تدار بطريقة جماعية وتتلقى دعاً عالمياً،تستخدم في تاطير الاساتذة الجلدد على مستوى الجامعات الجزائرية لتمكينهم من التو اصل و التفاعل مع الطلبة و المدرسين بعد ايداع محاضر اتهم ·

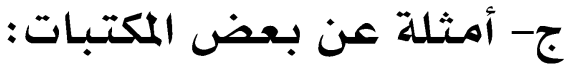

أتاحت بعض دور النشر والمطابع ومتاجر الكتب والمكتبات العامة كتبها الإلكترونية بجانا لمساعدة القراء على الاستفادة من أوقاتهم التي يمضونها في الحجر المنزلي تجنبا لعدوى الجائحة، وفيا يلي بعض هذه الموارد

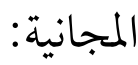

أمازون : أتاحت شركة أمازون -أكبر متجر كتب إلكتروني في العالم- ججموعة كبيرة من الكتب المسموعة بلغات عدة للفئات العمرية المختلفة بجانا، ويمكن الوصول إلى هذه المكتبة الصوتية الكبيرة عبر الرابط: https://stories.audible.com/start-listen مكتبة الإسكندرية : بالتزامن مع أغلاق أبوابها أمام الجممهور ضمن إجراءات السلامة والوقاية من الجائحة، طرحت مكتبة الإسكندرية كتبها الإلكترونية كلها بجانا للزوار عبر موقعها الإلكتروني على الإنترنت: https://www.bibalex.org/ar

رد شلف : اشتهرت المنصة -التي تصنف كواحدة من أسرع الشركات الخاصة نموا في الولايات المتحدة- بتوفير المواد الرقمية لملايين الطلاب عبر آلاف الجامعات، ويوفر موقعها الإلكتروني في الوقت الحالي عددا كبيرا من الكتب الإلكترونية ججانا بعد أن اتفق مع شركات النشر على تسهيل الوصول إليها /https://www.about.redshelf.com : في ظل الجائحة هارفرد بيزنس ريفيو : اشتهر الموقع بخدماته ومقالاته ومقاطعه المرئية التي تقدم نصائح إدارية وفي جالات التنمية والتطوير والأعلال، ومنح الموقع اشتراكا بجانيا يتضمن إرسال مقالات ومقاطع فيديو يومية كانت محصورة بالمشتر كين: /https://dash.harvard.edu المكتبة الوطنية الفرنسية ملايين المصادر والمراجع ججانا تحت تصرفكم مع إمكانية التحميل: https://gallica.bnf.fr/conseils/content/epub ، https://gallica.bnf.fr 
جامعة نيويورك أبو ظبي المجموعات العربية على الانترنت: وهي عبارة عن مكتبة عامة رقميَّة توفّر إمكانيّة الولوج الإلكتروني إلى أكثر من 13236 كتابا باللغة العربية. المكتبة الرقمية العالمية: /https://www.wdl.org/fr موقع أوكسفورد برس : للمطبعة الجامعية أنه أتاح موارده حول الفيروسات التاجية بجانا لمساعدة الباحثين والمختصين وحتى صانعي السياسات وغيرهم من يعملون على معالجة الوباء، بها في ذلك أكثر من 2500 مقالة بحثية وفصول عبر الإنترنت ذات صلة بالفيروس، وأعلن كذلك إتاحة وصول بجاني مؤقت لكتبه الإلكترونية عبر منصة رد شلف: /https://global.oup.com دار نشر بريل: أتاحت دار النشر العريقة كتبا ومقالات بحثية وعلمية عديدة حول مواضيع مثل الصحة العامة والتعليم عن بعد وأبحاث الأزمات، وتقوم بإضافة المحتوى الجمديد وتتيحه بجانا للزوار في الوقت /https://brill.com الحالي:

فرضت جائحة فيروس كورونا المستجد (كوفيد-19 ) تحديات لم يسبق لها مثيل منذ الحرب العالمية الثانية (1939-1945 ) على دول العالم كافة، أثرت في جوانب الحياة كلها، على غرار التعليم هو الاخر الذي تأثر من سياسة الاغلاق التام ، ومع الحرص على تجنّب تعليق الدراسة لجات جل حكومات الدول الى التعليم التفاعلي بدلا من التلقيني بامتياز لشتّى تطبيقات البَرامِج الإلكترونيّة مما خصب المجال أمام المكتبات الإلكترونية التي فتحت أبوابها أمام الطلبة والدارسين لتحميل محاضراتها، دروسها وموادها التعليمية بمختلف انو اعها ، كتبها في زمن جائحة كورونا من خلال إتاحة الخدمات الرقمية على مدار الساعة طو ال أيام الأسبوع وذلك بتوفير الكتب الإلكترونية والكتب الصوتية والمجلات والأفلام . ناهيك عن إمكانية الولوج إلى فهارس تلك المكتبات ومصادر معلوماتها الإلكترونية وعبر مختلف خدماتها المرجعية الافتراضية ومنصات التواصل الاجتماعي وموقع المكتبة الإلكتروني بالاضافة الم عقد وحضور 
الندوات والمؤتمرات وورش العمل عبر الإنترنت التي تقدمها الجامعات في شتى الموضوعات، علاوة على التواصل المستمر بين كثير من المكتبات وناشري وموردي الكتب وغيرها من مصادر المعلومات، لاختيار واستلام وإعداد المناسب منها لتلبية احتياجات بجتمع المستفيدين، بل بادرت بعض المكتبات بتوفير خدمة تو صيل الكتب إلى المنازل معقمة ومغلفة تلبية لرغبة مستفيديها، وإتاحة إمكانية ردها في أماكن مخصصة لذلك خارج مبنى المكتبة كل هذا من خلال اتمتة ادارة المكتبات التي تصدت لجائحة كوفيد 19 وضمان لاستجابة استمرارية التعليم بعيدا عن الشروط المادية من طاولات ومقاعد واستبدالها بموارد الكترونية، الأمر الذي عززته وأوصت به اليونسكو من خلال اطلاق منصات إلكترونية موضوعية توفر محتويات تعليميّة مفتوحة، ومساحات إلكترونية للتعلّم تتيح للطالب الحصول على الدعم الذي يتناسب مع الظروف الراهنة.

L'organisation automatisée de la bibliothèque de l'institut universitaire européen de Florence dans la perspective du service bibliothécaire national italien, tome 24, ${ }^{\circ} 5$, mai 1979 , Bultetin des bibliothèques de France,Tome ,1981,Nº1, p.11-25 .Disponible en ligne : http://bbf.enssib.fr/consulter/bbf-1981-01-0011002:ISSN 1292-8399. (2) عامر ابراهيم قتديلجي و إيمان فاضل السامرائي، حوسبة (أتمتة) المكتبات، ص 24، دار المار الميسرة، 2010، 2010، الأردن.

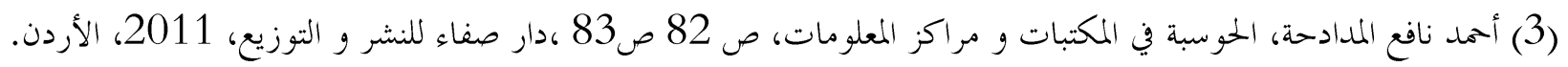

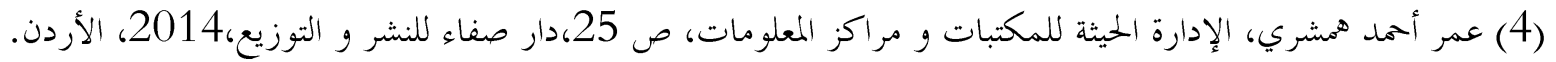
(5) جمال بدير، علم المكتبات و مراكز المعلومات، ص 78 78،دار الحامد للنشر و التوزيع، 2008 ، الأردن.

$$
\begin{aligned}
& \text { (6) عمر أحمد همري، مرجع سابق، ص } 25 \text { ص } 26 .
\end{aligned}
$$

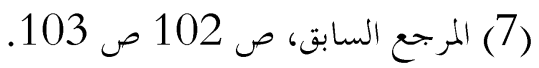

$$
\begin{aligned}
& \text { (8) مرجع نفسه، ص } 135 \text { ص } 136 .
\end{aligned}
$$

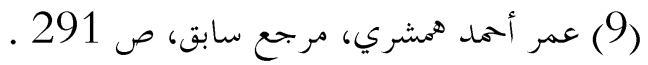

(10) ربحي مصطفى عليان، إدارة المكتبات الأسس و العمليات، ص صن 183، 183دار صفاء للطباعة و النشر و التوزيع، 2009، الأردن. (11) ربحي مصطفى عليان، ادارة و تنظيم المكتبات و مصادر التعلم، ص 168 ، دار صفاء للطباعة و النشر و التوزيع، 2002، لانيات الاردن. 


$$
\begin{aligned}
& \text { (12) مرجع نفسه، ص } 168 \text {. }
\end{aligned}
$$

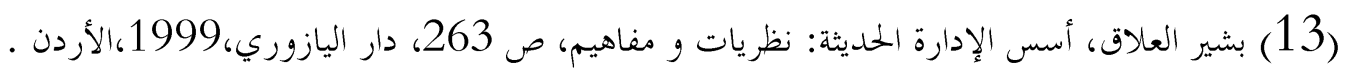

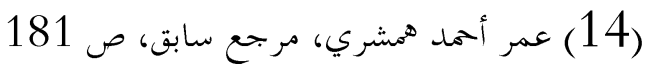

$$
\begin{aligned}
& \text { (15) عحمد الجيوسي، الإدارة علم و تطبيق، ص } 128 \text { ص } 129 \text { ص } 129 \text { ، دار الميسرة، 2000، الأردن. }
\end{aligned}
$$

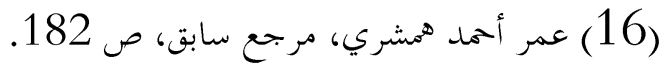

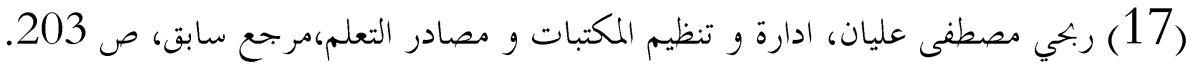

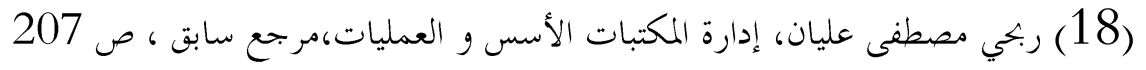

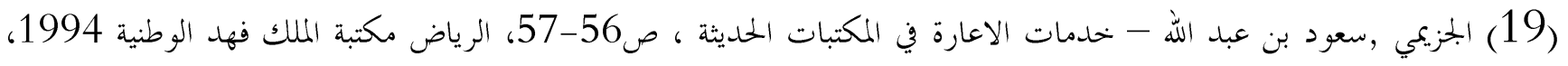

$$
\text { المملكة العربية السعودية. }
$$

DUCHEMIN, Pierre Yves - L'art d'informatiser une bibliothèque : Guide pratique. p.19, éd. du (20) cercle de la librairie, 1996, Paris.

SANDERS. DONALD-H. Informatique : un instrument de la gestion., p23, Mc Graw-Hill. 1980 (21) , Quebèc.

DUCHEMIN, Pierre-Yves. opcit. p. 21 (22)

(23) بدر أحمد،المكتبات المتخصصة ومراكز المعلومات في إدارة وتنظيم المعلومات،صاصنا،156،المكتبة الأكاديمة،1988، ،القاهرة. (24) عبد الهادي محمد فتحي ،المواد غير المطبوعة في المكتبات الشاملة، ص 146 ، الدار المصرية اللبنانية، 1994، 1994، القاهرة.

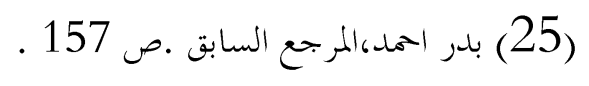

(26) عمر أحمد همشري و ربكي مصطفى عليان، أساسيات علم المكتبات و التوثيق و المعلومات، ص لماتع 143، مديرية المكتبات و الوثائق

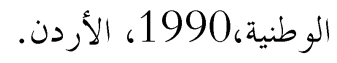

2019/03/30 http://www.webreview.dz/IMG/pdf/technologie.pdf (27) (28) ربحي مصطفى عليان و يسرى أبو عجينة، تنمية بمموعات المكتبة، ص 145، دار صفاء للنشر و التوزيع، 2000، الأردن.

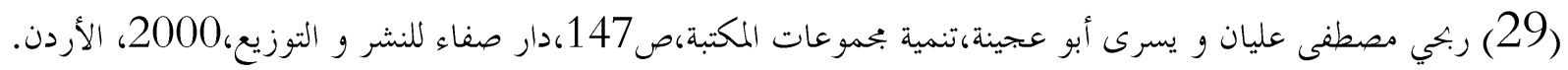
(30) عمر أحمد همشري، المكتبة و مهارات استخدامها، ص 173 ،دار صفاء لنشر و التوزيع، 2009، الأردن.

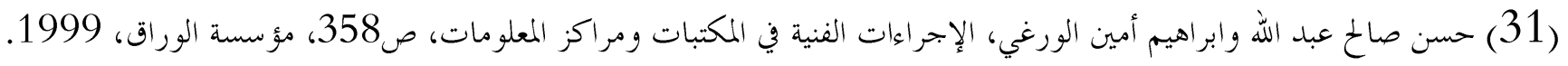
(32) فاطمة شباب، الفهارس الآلية المتاحة عبر شبكة الأنترنت : دراسة مسحية تقويمية لفهارس مكتبات مؤسسات التعليم العالي على إنى ضوء إرشادات الإفلا (IFLA) ، ص18 ص 19، مذكرة ماجستير:علم المكتبات و التوثيق ، 2008. الجزائر.

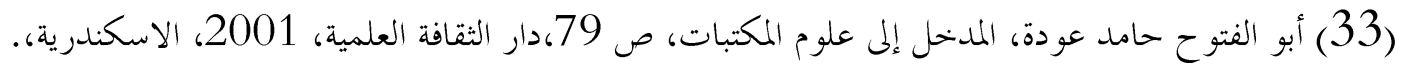


(34) العياشي بدر الدين، خدمات أنظمة المعلومات الإلكترونية ودورها في تلبية احتياجات المستفيدين: دراسة ميدانية بمكتبة المدرسة

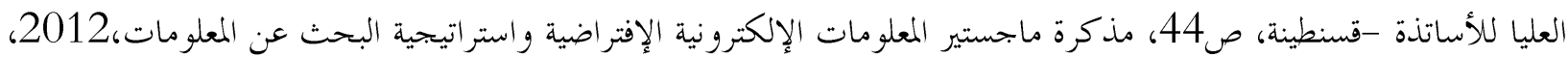

$$
\text { قسنطينة. }
$$

(36) محمد فتحي عبد الهادي وآخرون، التكشيف والاستخلاص: المفاهيم،الأسس،التطبيقات، ص 21، الدار المصرية اللبنانية، 2000. (37) عبد الحافظ محمد سلامة، خحدمات المعلومات و تنمية المقتنيات المكتبية، ص 69،دار الفكر للطباعة، 1997، عمان. (38) العياشي بلدر الدين، مرجع سابق ص 43. (39) بدر أحمد، التكشيف و الاستخلاص، دراسات في التحليل الموضوعي، ص 166،دار قباء، 2001، القاهرة.

$$
\text { (40) العياشي بدر الدين، مرجع سابق ، ص } 43 \text { (40) }
$$$$
\text { (41) ربكي مصطفى عليان، خحمات المعلومات، ص 61، دار صفاء للنشر و التوزيع، 2010، الأردن. }
$$$$
\text { (42) جعفر الجاسم، تكنولوجيا المعلومات، ص 153، دار أسامة، 2005، الأردن. }
$$$$
\text { (43) مرجع نفسه، ص } 153 \text { (14) }
$$

$$
\text { (44) ربكي مصطفى عليان، خحمات المعلومات، ص } 346 \text { ص ص } 347 .
$$

(45) غالب عوض النوايسة، خدمات المستفيدين في المكتبات ومراكز المعلومات، ص 165،دار صفاء للنشر والتوزيع، 2000،

$$
\text { الأردن. }
$$

(46) خراشية صليحة وعقريش مروة، تكنولوجيا المعلومات ودورها في احداث التغيير التنظيمي في المكتبات الجحامعية، ص 40،مذكرة

$$
\begin{aligned}
& \text { الماستر تخصص علم المكتبات، قالمة، 2017، الجزائر. } \\
& \text { (47) المرجع السابق، ص } 40 .
\end{aligned}
$$

2019/03/30 تاريخ الزيارة http://www.webreview.dz/IMG/pdf/technologie.pdf (48) (49) عامر ابر اهيم قندحلي و إيمان فاضل السامرائي، حوسبة (أتمتة) المكتبات، ص 206، دار الميسرة، 2010، الأردن.

$$
\begin{aligned}
& \text { (50) خراشية صليحة و عقريش مروة، مرجع سابق، ص } 41 . \\
& \text { (51) ربكي مطفى عليان، خحمات المعلومات، ص صر } 187 \text { ص } 188 . \\
& \text { (52) خراشية صليحة و عقريش مروة، مرجع سابق، ص } 42 .
\end{aligned}
$$

: 25/05/2020. https://ar.unesco.org/covid19/educationresponse (53)

: 23 https://ar.unesco.org/hemes/education-emergencies/coronavirus-school-closures/nationalresponses (54)

$$
\text { mars } 2020
$$


21 avr. ، https://ar.unesco.org/themes/education-emergencies/coronavirus-school-closures/solutions 